\title{
Twisted scroll waves organize Dictyostelium mucoroides slugs
}

\author{
Dirk Dormann, Cornelis Weijer and Florian Siegert* \\ Zoologisches Institut, Universität München, Luisenstrasse 14, 80333 München, Germany \\ *Author for correspondence (e-mail: si@zi.biologie.uni-muenchen.de)
}

\section{SUMMARY}

Cellular slime moulds (Dictyosteloids) are characterised by at least two different modes of slug migration. Most species, e.g. Dictyostelium mucoroides, produce a stalk continuously during slug migration, while a few species, e.g. Dictyostelium discoideum are characterised by stalk-less slug migration and only produce a stalk upon culmination. Experiments on $D$. discoideum and theoretical model calculations have shown that $D$. discoideum slugs are organized by a cAMP scroll wave in the tip which produces planar waves in the back. These waves guide cell movement in slugs: spiralling in the tip and forward movement parallel to the slug axis in the back. Simple changes in model parameters can lead to the formation of a twisted scroll wave which extends throughout the slug. In order to investigate whether such twisted scroll waves occur naturally we have analysed the movement of fluorescently labelled single cells in migrating $D$. mucoroides slugs. The results show that cells in the prespore zone of $D$. mucoroides slugs move in a spiral path. Although the velocity of single cells in $D$. mucoroides is faster than in $D$. discoideum, the net forward component of their movement is less due to their spiral trajectories. As a result $D$. mucoroides slugs move more slowly than $D$. discoideum slugs. The entire $D$. mucoroides slug also describes a spiralling path leaving corkscrew shaped stalks behind. Based on these observations we propose that cell movement in $D$. mucoroides slugs is controlled by a propagating twisted scroll wave of cAMP which extends throughout the length of the slug.

Key words: Morphogenesis, Slug, Excitable system, Chemotaxis, Scroll wave, Dictyostelium mucoroides

\section{INTRODUCTION}

The cellular slime moulds are an excellent model system to investigate a wide array of biological processes including biological oscillations, cellular communication, signal transduction, cell differentiation and the cellular basis of morphogenesis. Morphogenesis in slime moulds results from differentiation of one cell type, the vegetative amoebae, into stalk and spore cells and involves extensive chemotactic cell movement to get the different cell types into the right place in the fruiting body.

All cellular slime mould species are characterised by a life cycle in which phases of vegetative growth alternate with phases of morphogenetic activity. For the free-living amoebae of Dictyostelium discoideum depletion of nutrients is the signal to initiate multicellular development. Aggregation is mediated by extracellular cAMP signals (Devreotes, 1989). The aggregation centre produces periodic cAMP pulses which are detected, amplified and relayed by surrounding cells leading to the formation of outward propagating cAMP spiral waves. Up to the mound stage cAMP wave propagation can be seen as optical density waves using darkfield optics and digital image processing techniques (Gross et al., 1976; Siegert and Weijer, 1995). These optical density waves are correlated with shape changes which cells undergo upon stimulation with cAMP (Alcantara and Monk, 1974). Although optical density waves are no longer visible at later stages, many experimental results indicate that extracellular cAMP controls the movement of cells in slugs and culminating fruiting bodies (Durston and
Vork, 1979; Siegert and Weijer, 1991; Reymond et al., 1995; Chen et al., 1996).

There is a characteristic pattern of cell movement in $D$. discoideum slugs: cells in the prestalk zone show vigorous rotational movement around the central core of the tip, while cells in the prespore zone move straight forward in the direction of slug migration (Siegert and Weijer, 1992). From these observations the geometry of the propagating signal was deduced: a three-dimensional scroll wave (spiral wave) produces rotational cell movement in the tip and planar wave fronts produce forward movement parallel to the slug axis in the prespore zone. Computer simulations using the Martiel-Goldbeter model of cAMP relay showed that conversion of a scroll wave into a series of planar waves occurs if there is a substantial difference in excitability between the prestalk and prespore cell population or if only a part of the prespore population actively relays the cAMP signal (Bretschneider et al., 1995; Steinbock et al., 1993). These simulations showed furthermore that a twisted scroll wave extends from the tip to the rear of the slug if the difference in excitability between prestalk and prespore cells is small or if a high number of the cells in the prespore zone relay the signal. Under these conditions the core of the spiral extends from the tip to the rear of the slug. These results suggested that Dictyostelium species which form a stalk during slug migration (Brefeld, 1869; Bonner, 1982) might be organized by a twisted scroll wave.

Despite the complex three-dimensional morphology and temporal variation of the cAMP signal, there is a stable well 
defined pattern of average cAMP concentrations over time in a slug: the cAMP concentration is high in the peripheral layer of the prestalk zone, lower in the prespore zone and extremely low in a central funnel in the tip (Bretschneider et al., 1995). This pattern coincides in $D$. discoideum with the cAMP requirements for differentiation into the different types of prestalk cells and prespore cells: differentiation of prestalk A cells requires high levels of external cAMP while their final differentiation into prestalk $\mathrm{AB}$ cells in the central funnel of the tip is triggered by low levels of external cAMP (Berks and Kay, 1990)

To test the prediction that a twisted scroll wave organizes $D$. mucoroides slugs we investigated cell movement patterns. This species behaves essentially the same as $D$. discoideum during the early stages of development (Raper, 1984). It also uses cAMP as a chemotactic signal during aggregation and optical density waves are visible up to the early mound stage (Bonner et al., 1972; Mullens and Newell, 1978; own unpublished observations). The major morphological difference between $D$. discoideum and $D$. mucoroides occurs during the slug stage: $D$. mucoroides slugs continuously form a stalk while migrating whereas D. discoideum slugs do not (Arndt, 1937).

\section{MATERIAL AND METHODS}

\section{Cell culture and labelling}

Wild-type Dictyostelium mucoroides cells were grown in association with Klebsiella aerogenes on SB-agar plates at $22^{\circ} \mathrm{C}$ (Sussman, 1987). After 40 to 48 hours the bacterial lawn had cleared and the cells were harvested with a spatula. The amoebae were resuspended in KK2 buffer $\left(20 \mathrm{mM} \mathrm{KH} 2 \mathrm{PO}_{4} / \mathrm{K}_{2} \mathrm{HPO}_{4}\right.$, pH 6.8) and washed five times with $\mathrm{KK} 2$ to remove bacteria.

For labelling with the vital dye Neutral Red $30 \mu 1$ of a $0.1 \%$ Neutral Red solution (dissolved in KK2) were added per $1 \times 10^{7}$ cells. After 1 minute of incubation cells were washed twice in distilled water, density was adjusted to $2 \times 10^{8}$ cells $/ \mathrm{ml}$ and small droplets $(1-2 \mu \mathrm{l})$ were placed on $1 \%$ water agar plates (1\% Difco Bacto-Agar in distilled water). Plates were incubated at $18^{\circ} \mathrm{C}$ in boxes with a single slit to allow light entry (Jermyn and Williams, 1991).

Two different methods were used to label cells with fluorescent dyes. For filter loading $8 \times 10^{7}$ cells were resuspended in $1 \mathrm{ml}$ of 10 $\mathrm{mg} / \mathrm{ml}$ FITC-Dextran (MW $20 \mathrm{kDa}$, Sigma) and pressed through a nylon membrane (Nytal HD-7 $\mu \mathrm{m}$, Schweiz. Seidengazefabrik Thal). About $1 \%$ of the cells were labelled by this procedure (Siegert and Weijer, 1991). Alternatively, cells were stained with 5-chloromethylfluorescein diacetate (CMFDA Cell Tracker Green, Molecular Probes, Inc.). Then $1 \times 10^{7}$ cells were incubated in $1 \mathrm{ml} 10 \mu \mathrm{M}$ CMFDA for 1 hour at room temperature (Knecht and Shelden, 1995). Cells were washed twice with KK2 and mixed with unlabeled cells to a final concentration of 5\% stained cells. After both labelling steps cells were washed twice in distilled water and treated as described above.

\section{Video microscopy}

Slugs were observed on a Zeiss Axiovert microscope equipped with $\times 5, \times 10$ and $\times 20$ Zeiss Plan-Neofluar objectives. Usually, slugs were filmed from below through the agar; for side views an agar block containing the slug was cut out and turned on its side as described by Dormann et al. (1996). To reduce light scattering on the slug surface during fluorescence microscopy slugs were submerged in mineral oil (Siegert and Weijer, 1992).

A monochrome CCD video camera (Sanyo, VC-2512) was used for bright field recordings. The video signal was digitised with an AFG frame grabber (Imaging Technology, Inc.), contrast enhanced and averaged to reduce noise. Images were acquired at intervals of 10 or
20 seconds and stored on an analogue laser video disc (SONY LVR4000P). Fluorescence imaging was done with a cooled CCD camera (Hamamatsu, C4880), that was digitally connected to the AFG and controlled by the HiPic software (Hamamatsu, Version 4.0). Time series were stored on hard disc and transferred to video disc afterwards. A computer controlled shutter blocked the excitation light after exposure. The light intensity of the $100 \mathrm{~W}$ mercury arc lamp was reduced to $10-50 \%$ with neutral density filters.

\section{Data analysis and image processing}

Labelled cells and slug tips were tracked manually from the recorded sequences on a video monitor, using an OFG frame grabber (Imaging Technology, Inc.) and custom software (Siegert and Weijer, 1992). Optical-density waves in slugs were visualised by image subtraction (Siegert and Weijer, 1995). Image subtraction reveals differences in successive images, since structures that do not change exhibit a uniform grey level while the moving structures appear as black and white bands. A gradient method was used to detect cell flow in slugs (Siegert et al., 1994). Vector fields were calculated that described the average movement at every pixel over a short sequence of 16 images. Resulting vectors were averaged to reduce noise and to improve visibility. The vectors indicate both direction and velocity of cell movement. Velocity was encoded by the length of the vector.

\section{RESULTS}

\section{D. mucoroides slugs show spiral cell movement}

Visual inspection of $D$. mucoroides slugs migrating on a water agar surface shows that the stalk extends from the tip through the whole body of the slug and is left behind during migration. In Fig. 1A several slugs have migrated from the point of aggregation towards a light source on the left. The stalks were often slightly twisted, an effect which was even more pronounced in the slug itself. In Fig. 1B the stalk is twisted along its whole length of more than $5 \mathrm{~mm}$. If slugs are kept under conditions favourable for migration, stalks can be up to several centimetres long. Fig. 1C shows a close up view (from bottom) of a migrating slug. Even within the slug body the stalk is twisted in many cases; in rare cases the slugs were so strongly deformed that the twists appeared like beads on a string. Fig. $1 \mathrm{D}, \mathrm{E}$ show the results of computer simulations described in detail by Bretschneider et al. (1995). The shaded grey tones in $\mathrm{D}$ depict the cAMP wave front. The prestalk zone to the left is organised by a scroll wave, which becomes twisted at the border between prestalk and prespore cells. The twisting occurs because the prestalk cells are assumed to be more excitable than the prespore cells. The less excitable prespore cells cannot relay the signal as fast as it is produced by the prestalk cells resulting in slower wave propagation in the prespore zone and hence twisting of the spiral. The red colour indicates the core of the twisted spiral, which is defined as the site of extremely low extracellular cAMP concentration. In E the shaded grey indicates the outer border of the slug, while the red colour indicates the location and shape of the core of the twisted scroll wave. The appearance of the stalk in the computer simulation showed a remarkable similarity to stalks in real $D$. mucoroides slugs.

The analysis of cell movement in migrating slugs showed that the cells did not move straight forward parallel to the direction of slug migration but followed a spiral path which wound around the stalk. Fig. 2A shows the anterior half of a typical slug. The prespore zone has several bulges which gives 
Fig. 1. Migration with stalk formation in Dictyostelium mucoroides. (A) Slugs migrating towards a light source on an agar surface. Bar, $500 \mu \mathrm{m}$. (B) A twisted stalk several millimeters long left behind during slug migration. Bar, $500 \mu \mathrm{m}$. (C) Close up view of a migrating slug. Note the twisted stalk in the interior of the slug.

(D) Computer simulation showing the cAMP wave front (shown as a grey shaded isosurface) and the core of the spiral (red).

(E) Computer simulation showing the region of constant low cAMP concentration (red) and the outer border of the slug cylinder.
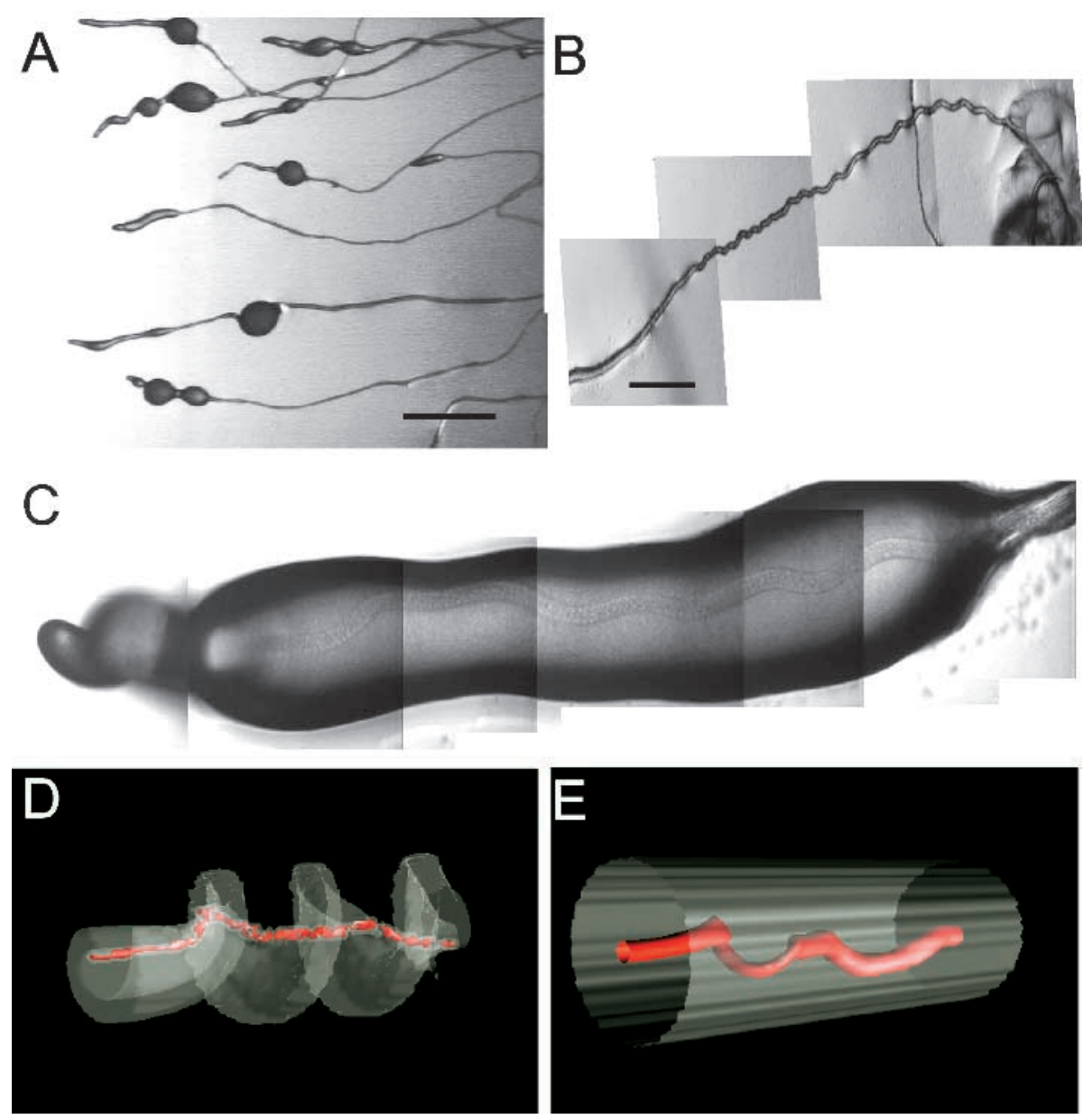

the slug a twisted appearance. The stalk is only slightly twisted. In order to quantitate cell movement we analysed time lapse videos of slugs, in which 1-5\% of the cells were fluorescently labelled with fluorescein dextran or Cell Tracker Green. Fig. 2B-E shows two adjacent sections of the slug in A (indicated by the squares in A) and the corresponding velocity vector fields. Velocity vector fields indicate the direction and speed (encoded as the length of the vectors) of cell movement. As can be seen in Fig. 2D,E the cells within the bulges moved at a steep angle to the direction of slug movement. Only within the intersections between bulges did cells move in the direction of slug migration (from left to right). Time lapse videos at low magnification showed that labelled cells followed a spiral path along the slug axis except in the tip region where they showed clear rotational movement as also observed in D. discoideum. These observations suggest that a twisted scroll wave of cAMP originating from the tip organises cell movement. The scroll wave causes rotational cell movement in the tip and serves as a pacemaker for the prespore cells. At the border between the prestalk and prespore zone the scroll wave transforms into a twisted scroll wave leading to the observed spiral cell movement in the prespore zone.

\section{Movement speed of slugs and single cells in the prespore zone}

Table 1 shows that $D$. mucoroides slugs move with half the speed of $D$. discoideum slugs. A similar value for $D$. mucoroides slug migration was published by Samuel (1961). To determine whether this slower slug speed was caused by slower cell movement we measured the speed of single fluorescently labelled cells in the prespore zone. As can be seen in Table 1,D. mucoroides cells move significantly faster than $D$. discoideum cells. Thus the slower speed of $D$. mucoroides slugs must be due to the lesser efficiency of spiral cell movement in producing forward movement.

\section{Periodic tip movement}

The hypothesis that a twisted scroll wave organises $D$. mисоroides slugs requires oscillatory signalling and chemotactic cell movement. Therefore we searched for periodic events during the migration of $D$. mucoroides slugs. Time lapse videos of migrating slugs showed that the tip undergoes periodic velocity changes. Fig. 3A shows an extremely long slug whose movement was filmed over a period of more than

Table 1. Comparison of slug and cell movement speed

\begin{tabular}{|c|c|c|}
\hline & Slug movement & Cell movement \\
\hline D. mucoroides & $\begin{array}{c}9.6 \mu \mathrm{m} / \text { minute } \pm 3.2 \\
n=20\end{array}$ & $\underset{n=70}{22.3 \mu \mathrm{m} / \text { minute }} \pm 5.4$ \\
\hline D. discoideum & $\begin{array}{c}16.7 \mu \mathrm{m} / \text { minute } \pm 5.7 * \\
\text { anterior-like }+ \text { psp cells }\end{array}$ & $\begin{array}{c}17.3 \mu \mathrm{m} / \text { minute } \\
\text { psp cells }\end{array}$ \\
\hline
\end{tabular}


Fig. 2. Movement of fluorescently labelled cells in a D. mucoroides slug. (A) Brightfield image of the anterior $2 / 3$ of a migrating slug. Bar, $100 \mu \mathrm{m}$. $(B, C)$ The same slug as in A viewed under excitation light to visualize cells labelled with Cell Tracker Green. The black rectangles in A indicate the location of the photographs.

$(\mathrm{D}, \mathrm{E})$ Velocity vector fields of $\mathrm{B}$ and $\mathrm{C}$ obtained by calculating the

displacement of the bright pixels over a series of 16 successive images (160 seconds). The length of the vectors indicates movement speed.
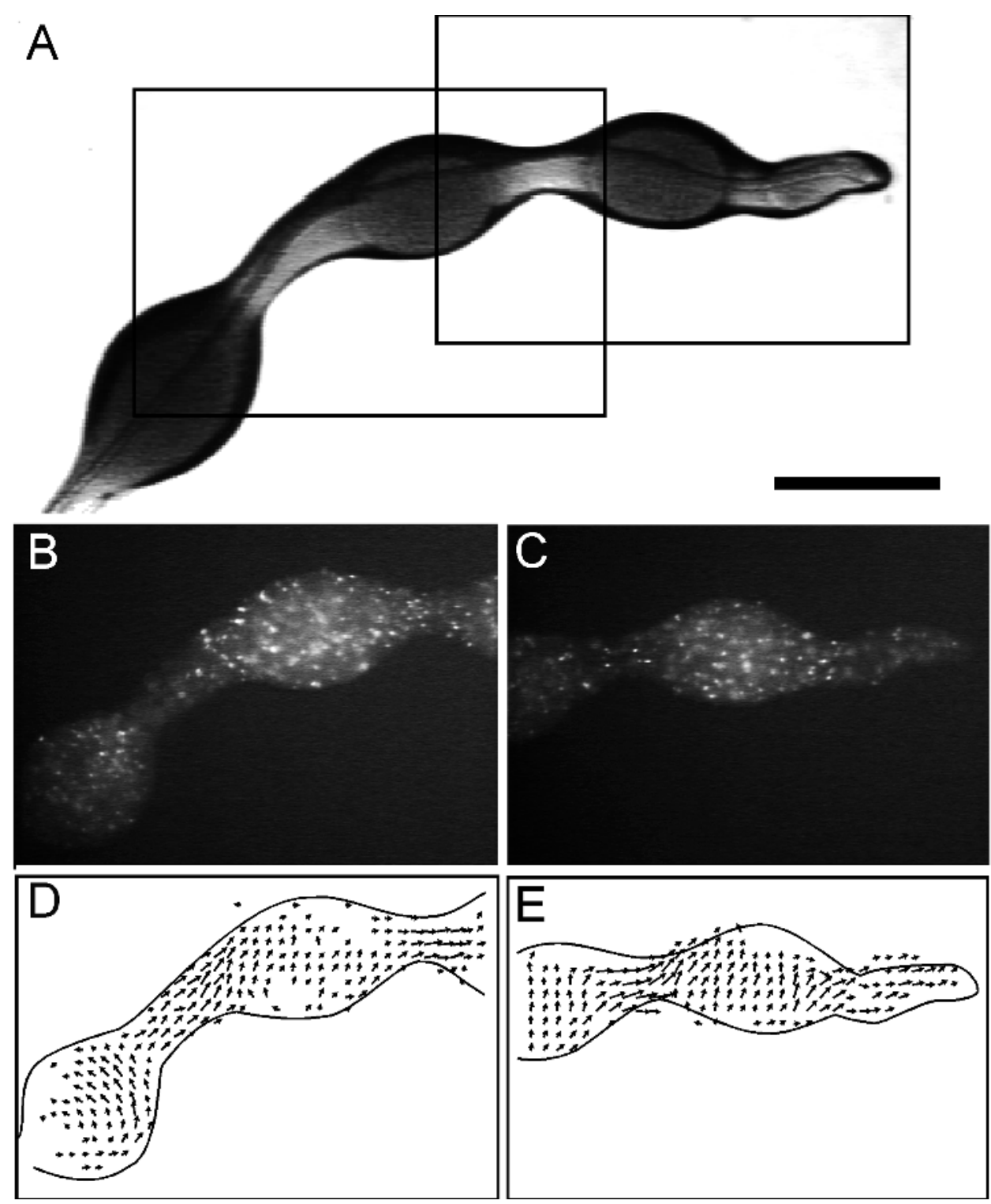

20 minutes. Periodic tip movement was quantitated by timespace plots (Siegert and Weijer, 1995). Fig. 3B shows the most anterior part of the tip. The window indicates the position of the line of pixels which was stored for successive time points. After the end of the experiment these lines were displayed below each other resulting in a time-space plot (Fig. 3C). The dark grey area on the right represents the migrating slug. Movement of the tip to the left is shown as the dark area advances from top to bottom in the time-space plot. The straight edge of the dark area indicates that slug movement was constant over time. The slope represents the movement velocity of the slug. Superimposed on this straight edge is a periodic signal which indicates periodic fast advances of the tip. Fig. 3D shows translocation of the tip recorded at 10 second intervals. Tip movement shows a clear periodicity of 2.2 minutes.

\section{Darkfield waves in the rear of slugs}

In about $10 \%$ of all slugs investigated we observed optical density waves in the most posterior part of the prespore region. These waves represent faint modulations of the optical density of the cell stream and appeared to be correlated with shape changes which cells undergo upon chemotactic stimulation. Fig. 3E shows an enlargement of the area indicated by the black square in Fig. 3A. We used time-space plots to investigate the periodicity and propagation velocity of the faint optical density waves in this part of the slug. Fig. 3F shows the time-space plot. The optical density waves appear as slightly tilted horizontal stripes which propagate from left to right. From the distance we derived a period length of successive waves of $4.03 \pm 1.39$ minutes $(n=15)$. From the slope we derived an average propagation speed of the waves of $79.8 \mu \mathrm{m} /$ minute \pm 25 $(n=16)$. These values correspond well with measurements of cell movement in mounds of $D$. discoideum (Siegert and Weijer, 1995). At the lower right corner of Fig. 3F one can see stripe patterns oriented opposite to the optical density waves (propagating from right to left). These are due to cell movement in the direction of slug movement and optical 
Fig. 3. Periodic events in a $D$. mucoroides slug. (A) An exceptionally long slug in which both periodic tip movement and wave propagation could be measured. Bar, $200 \mu \mathrm{m}$. (B) The foremost part of the tip. The black rectangle indicates the location of the window used for the construction of the time-space plot. (C) Time-space plot. (D) Rate of tip movement as determined by plotting the displacement of the tip versus time. (E) Enlargement of the thin, stream-like part of the slug indicated by the black rectangle to the right in A. (F) Time-space plot. (G) Subtraction image prepared from $\mathrm{E}$ and two successive images taken at 10 second intervals.
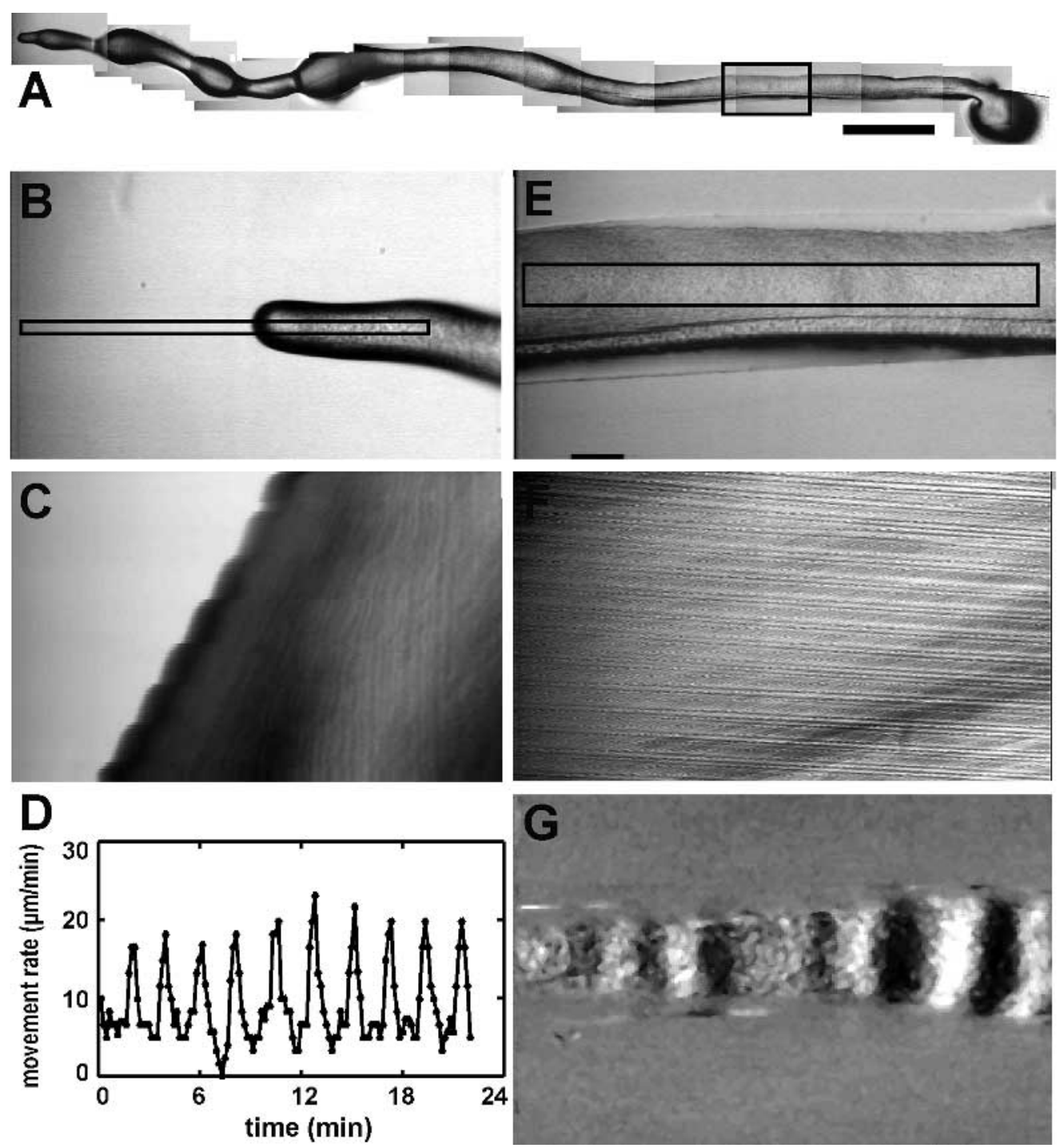

density wave propagation. Measurements of the slope of these waves yielded an average cell movement speed of 18 $\mu \mathrm{m} / \mathrm{minute} \pm 2.9(n=13)$. To visualise the optical density waves in Fig. 3G more clearly we used an image subtraction algorithm. Often these waves travelled in pairs of two or three dark bands and their number seemed to increase towards the rear of the slug. Our impression is that these groups of bands represent cell shape changes to just one passing cAMP signal.

\section{A twisted scroll wave can convert into planar waves}

The observation of planar wave fronts in $D$. mucoroides slugs is not consistent with the hypothesis that a twisted scroll organises cell movement in D. mucoroides. However we observed optical density waves only in slugs which were extremely elongated and had unusually flat prespore zones. Normal slugs are about 50 to 100 cell diameters thick. Slugs, in which we could measure optical density waves, were only 3-8 cell diameters thick and resembled in this respect aggregation streams. We performed computer simulations using the model and parameter sets described by Bretschneider et al. (1995) in order to explore the possibility that a reduction in slug diameter result in the formation of planar waves. We chose conditions which initiated a twisted scroll wave in a slug of 40 cells diameter and observed what happened if this twisted scroll wave propagated into a domain of only 4 cells diameter. As shown in Fig. 4 a reduction in slug diameter causes the formation of planar waves from a spiral wave. In the domain where the slug is 40 cell diameters thick the wave propagates as a twisted scroll. However, as this wave propagates into the thin domain the scroll cannot be propagated and a series of planar waves forms.

\section{DISCUSSION}

\section{Oscillatory behaviour in slugs}

If $D$. mucoroides slugs are organised by chemotactic signals and wave propagation, it should be possible to observe periodic cell behaviour. cAMP stimulated cells move towards increasing chemoattractant concentrations and stop moving when a wave front has passed (Alcantara and Monk, 1974; Devreotes, 1989). We found clear evidence for periodic events in migrating $D$. mucoroides slugs. The tip underwent periodic movements at an average period of about 3 minutes similar to 
Fig. 4. Computer simulation of wave propagation in a $D$. mucoroides slug. (A) Brightfield image of a slug with a long and thin tail. (B) Isosurface of a propagating cAMP wave.

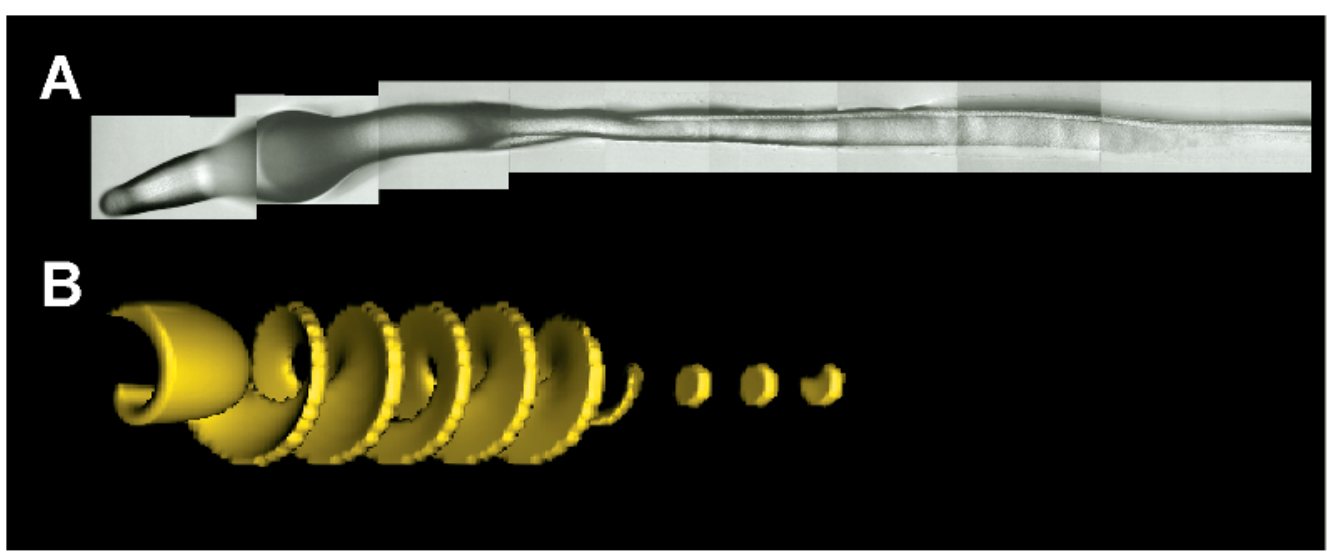

the periodic tip progression measured during the extension of culminates of D. discoideum (Durston and Vork, 1979; Durston et al., 1976). $D$ discoideum culmination and $D$. mucoroides slug migration are essentially similar events since they both involve movement of a mass of cells along a forming stalk. The cellular basis for the periodic tip extension (Fig. 3D) is unclear but could result from synchronised shape changes after passage of a cAMP wave or alternatively from the periodic extension of the stalk itself. However, it could also be caused by a gradual build up of tension due to cells adhering to the stalk, which is periodically released by a slippage of the cell mass along the stalk. Clarification of possible mechanisms has to await further experiments.

We also observed the propagation of optical density waves in the back of $D$. mucoroides slugs. In $D$. discoideum cAMP wave propagation can be seen as an optical density wave during early aggregation and in mounds (Rietdorf et al., 1996). The optical density waves represent the propagating cAMP signal (Tomchik and Devreotes, 1981). In D. discoideum it is not possible to see the waves anymore, most likely since the rear ends of slugs are to thick. In the thin posteriors of $D$. mucoroides slugs, however, they are clearly visible and show conclusively that slugs are organised by propagating waves of a chemoattractant.

\section{A twisted scroll wave organises D. mucoroides slugs}

Time-lapse video recordings of fluorescently labelled cells combined with single cell tracking and velocity vector analysis showed that single cell movement followed the path of a twisted scroll in all D. mucoroides slugs investigated. These cells do not move straight forward but describe a corkscrew forward motion around the stalk. Nevertheless overall slug movement is straight forward. Furthermore this peculiar type of cell movement led to a characteristic change in slug shape: many slugs were corkscrew shaped. This type of cell movement suggested that the underlying chemotactic signal is a twisted scroll wave. Obviously the twisted shape of the chemotactic signal can lead easily to a twisting of the whole cell mass.

In the mathematical model twisted scrolls occur if there is just a slight difference in the excitability between the prestalk and prespore cell population. If the prespore cells are less excitable than the prestalk cells the scroll in the tip becomes deformed when propagating into the less excitable prespore cells. A larger difference in excitability leads to the further twisting of the wave fronts until the twist is so strong that wave fronts break off and form planar waves in the prespore zone as in D. discoideum (Bretschneider et al., 1995). There is reason to suppose that the difference in excitability between prestalk and prespore cells in D. mucoroides might not be as great as in $D$. discoideum, since there is a continuous re-differentiation from prespore to prestalk cells during the slug migration (Gregg and Davis, 1982). Up to $90 \%$ of the initial cell mass can be used for stalk formation. Cells from the prespore zone have to be able to migrate into the more excitable tip zone in order to replace differentiated stalk cells which are left behind in the stalk during migration (Bonner, 1982). This necessity for sorting and cell type conversion could imply that prespore and prestalk cell might differ only slightly in their chemotactic and relay properties.

We suggest that the prestalk cells actually relay less efficiently than in $D$. discoideum. This conjecture could explain two experimental facts: first, the presence of a scroll wave and second the premature formation of a stalk. A low excitability of the prestalk cells would reduce the difference in excitability between both cell types and therefore lead to a twisted scroll wave. Furthermore, a lower excitability in the prestalk zone would result in a lower average cAMP concentration in the core region of the tip thus favouring premature stalk differentiation.

\section{Migration with stalk formation versus stalk-less migration}

The slug stage enables cellular slime moulds to find an optimal location for fruiting body construction. $D$. discoideum slugs are sensitive to light, $\mathrm{pH}$, volatile chemicals and even slight differences in temperature (Bonner, 1994). These abilities allow them to migrate towards the surface of the litter stratum thus to have a better chance for spore dispersal. Comparison of different species shows that there are two basic modes of slug migration: migration with stalk formation and migration without stalk formation. Out of 50 known species of cellular slime moulds only four species show stalk-less migration (Bonner, 1982). The obvious advantage for stalk-less migration is that there is no loss of cells during migration. D. mucoroides slugs lose up to $95 \%$ of their cell mass during prolonged migration and fruiting body construction. During this process prestalk cells differentiate to stalk cells, die and have to be replaced by a re-differentiation of prespore cells. Prespore cells are therefore continuously lost thus reducing the effectivity of 
spore dispersal. In contrast $D$. discoideum loses relatively few cells during migration: usually $50-70 \%$ of all cells of an aggregate become spores. From these observations and the results presented in this paper it could be hypothesized that migration with stalk formation is the more primitive form, since it is less efficient and many cells are sacrificed.

We thank Dr C. N. David for carefully reading the manuscript, Dr J. T. Bonner for his helpful comments and T. Bretschneider for performing the model calculation. This work was supported by the Deutsche Forschungsgemeinschaft (We 1127).

\section{REFERENCES}

Alcantara, F. and Monk, M. (1974). Signal propagation during aggregation in the slime mould Dictyostelium discoideum. J. Gen. Microbiol. 85, 321-334.

Arndt, A. (1937). Rhizopodenstudien III. Untersuchungen uber Dictyostelium mucoroides Brefeld. W. R. Arch. Entw. Mech. Org. 136, 681-744.

Berks, M. and Kay, R. R. (1990). Combinatorial control of cell differentiation by cAMP and DIF-1 during development of Dictyostelium discoideum. Development 110, 977-984.

Bonner, J. T., Hall, E. M., Noller, S., Oleson, F. B. Jr and Roberts, A. B. (1972). Synthesis of cyclic AMP and phosphodiesterase in various species of cellular slime molds and its bearing on chemotaxis and differentiation. Dev. Biol. 29, 402-409.

Bonner, J. T. (1982). Evolutionary strategies and developmental constraints in the cellular slime molds. Am. Naturalist 119, 530-552.

Bonner, J. T. (1994). The migration stage of Dictyostelium: Behavior without muscles or nerves. FEMS Microbiol. Lett. 120, 1-2.

Brefeld, O. (1869). Dictyostelium mucoroides. Ein neuer Organismus aus der Verwandtschaft der Myxomyceten. Abhandlungen der Senckenbergischen Naturforschenden Gesellschaft Frankfurt 7, 85-107.

Bretschneider, T., Siegert, F. and Weijer, C. J. (1995). Three-dimensional scroll waves of cAMP could direct cell movement and gene expression in Dictyostelium slugs. Proc. Nat. Acad. Sci. USA 92, 4387-4391.

Chen, M. Y., Insall, R. H. and Devreotes, P. N. (1996). Signaling through chemoattractant receptors in Dictyostelium. Trends Genet. 12, 52-57.

Devreotes, P. (1989). Dictyostelium discoideum: A model system for cell-cell interactions in development. Science 245, 1054-1058.

Dormann, D., Siegert, F. and Weijer, C. J. (1996). Analysis of cell movement during the culmination phase of Dictyostelium development. Development 122, $761-769$

Durston, A. J., Cohen M. H., Drage D. J., Potel M. J., Robertson A. and Wonio, D. (1976). Periodic movements of Dictyostelium discoideum sorocarps. Dev. Biol. 52, 173-180.
Durston, A. J. and Vork, F. (1979). A cinamatographical study of the development of vitally stained Dictyostelium discoideum. J. Cell Sci. 36, 261-279.

Gregg, J. H. and Davis, R. W. (1982). Dynamics of cell redifferentiation in Dictyostelium mucoroides. Differentiation 21, 200-205.

Gross, J. D., Peacey, M. J. and Trevan, D. J. (1976). Signal emission and signal propagation during early aggregation in Dictyostelium discoideum. $J$. Cell Sci. 22, 645-656.

Jermyn, K. A. and Williams, J. G. (1991). An analysis of culmination in Dictyostelium using prestalk and stalk-specific cell autonomous markers. Development 111, 779-787.

Knecht, D. A. and Shelden, E. (1995). Three-dimensional localization of wildtype and myosin II mutant cells during morphogenesis of Dictyostelium. Dev. Biol. 170, 434-444.

Mullens, I. A. and Newell, P. C. (1978). cAMP binding to cell surface receptors of Dictyostelium. Differentiation 10, 171-176.

Raper, K. B. (1984). The Dictyostelids. Princeton, NJ: Princeton University Press.

Reymond, C. D., Schaap, P., Veron, M. and Williams, J. G. (1995). Dual role of cAMP during Dictyostelium development. Experientia 51, 1166-1174.

Rietdorf, J., Siegert, F. and Weijer, C. J. (1996). Analysis of optical density wave propagation and cell movement during mound formation in Dictyostelium discoideum. Dev. Biol. 177, 427-438.

Samuel, E. W. (1961). Orientation and rate of locomotion of individual amebas in the life cycle of the cellular slime mold Dictyostelium mucoroides. Dev Biol. 3, 317-335.

Siegert, F. and Weijer, C. J. (1991). Analysis of optical density wave propagation and cell movement in the cellular slime mold Dictyostelium discoideum. Physica D 49, 224-232.

Siegert, F. and Weijer, C. J. (1992). Three-dimensional scroll waves organize Dictyostelium slugs. Proc. Nat. Acad. Sci. USA 89, 6433-6437.

Siegert, F., Weijer, C. J., Nomura, A. and Miike, H. (1994). A gradient method for the quantitative analysis of cell movement and tissue flow and its application to the analysis of multicellular Dictyostelium development. $J$. Cell Sci. 107, 97-104.

Siegert, F. and Weijer, C. J. (1995). Spiral and concentric waves organize multicellular Dictyostelium mounds. Curr. Biol. 5, 937-943.

Steinbock, O., Siegert, F., Muller, S. C. and Weijer, C. J. (1993). Threedimensional waves of excitation during Dictyostelium morphogenesis. Proc. Nat. Acad. Sci. USA 90, 7332-7335.

Sussman, M. (1987). Cultivation and synchronous morphogenesis of Dictyostelium under controlled experimental conditions. Meth. Cell Biol. 28, 9-29.

Tomchik, K. J. and Devreotes, P. N. (1981). Adenosine 3',5'-monophosphate waves in Dictyostelium discoideum: A demonstration by isotope dilutionfluorography technique. Science 212, 443-446.

(Received 17 March 1997 - Accepted 4 June 1997) 